Asian Spine Journal Vol. 6, No. 4, pp 227 232, 2012

http://dx.doi.org/10.4184/asj.2012.6.4.227

\title{
The Efficacy and Persistence of Selective Nerve Root Block under Fluoroscopic Guidance for Cervical Radiculopathy
}

\author{
Jae-Yoon Chung, Ji-Hyeon Yim, Hyoung-Yeon Seo, Sung-Kyu Kim, Kyu-Jin Cho \\ Department of Orthopedic Surgery, Chonnam National University Hospital, \\ Chonnam National University Medical School, Gwangju, Korea
}

\begin{abstract}
Study Design: Retrospective study.
Objectives: To investigate the outcomes of fluoroscopically guided selective nerve root block as a nonsurgical treatment for cervical radiculopathy.

Overview of Literature: Only a few studies have addressed the efficacy and persistence of cervical nerve root block.

Methods: This retrospective study was conducted on 28 consecutive patients with radicular pain due to cervical disc disease or cervical spondylosis. Myelopathy was excluded. Cervical nerve root blocks were administered every 2 weeks, up to 3 times. Outcomes were measured by comparing visual analogue scale (VAS) scores, patient satisfaction, and medication usage before the procedure and at 1 week and 3, 6, and 12 months after the procedure. In addition, complications associated with the procedure and need for other treatments were evaluated.

Results: The average preoperative VAS score was 7.8 (range, 5 to 10), and this changed to 2.9 (range, 1 to 7 ) at 3 months and 4.6 (range, 2 to 7 ) at 12 months. Patient satisfaction was $71 \%$ at 3 months and $50 \%$ at 12 months. Five patients used medication at 3 months, whereas 13 used medication at 12 months. Average symptom free duration after the procedure was 7.8 months (range, 1 to 12 months). Two patients were treated surgically. Only two minor complications were noted; transient ptosis with Horner's syndrome and transient causalgia.

Conclusions: Although selective nerve root block for cervical radiculopathy is limited as a definitive treatment, it appears to be useful in terms of providing relief from radicular pain in about $50 \%$ of patients at 12 months.
\end{abstract}

Key Words: Cervical radiculopathy, Spinal nerve root, Nerve blocks

\section{Introduction}

Cervical radiculopathy causes disabling pain and is frequently encountered in spinal practice. Its annual average age-adjusted incidence is about 83.2 per 100,000 people [1]. Cervical radiculopathy can be caused by degenerative conditions such as cervical disc prolapse, but it is more commonly due to a combination of factors including disc herniation, osteoarthritis of uncovertebral and facet joints, decreased disc height and cervical spondylosis [1]. In terms of treatment, many patients improved on conservative treatment, and according to recent studies, symptoms were relieved by conservative treatment in up to $43 \%$ of patients [2]. On the other hand, some patients undergo surgery for severe pain and recurrence, but only moderate outcomes are achieved. In a multi-center study, up to $26 \%$ of patients were found to remain symptomatic after cervical surgery [3]. Therefore, selective nerve root block offers an alternative treatment when patients fail to respond to medication and conservative treatment. Furthermore, it may provide an

Received Nov 23, 2011; Revised Dec 30, 2011; Accepted Jan 4, 2012

Corresponding author: Ji-Hyeon Yim, MD

Department of Orthopedic Surgery, Chonnam National University Hospital, Chonnam National University Medical School, 42 Jebong-ro, Dong-gu, Gwangju 501-757, Korea

Tel: +82-62-220-6336, Fax: +82-62-225-7794, E-mail: presid50@naver.com

Copyright (C) 2012 by Korean Society of Spine Surgery

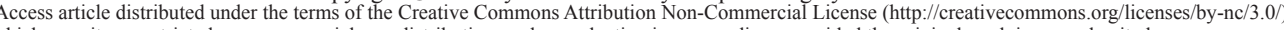
which permits unrestricted non-commercial use, distribution, and reproduction in any medium, provided the original work is properly cited. Asian Spine Journal • pISSN 1976-1902 eISSN 1976-7846 
effective alternative tool for those unwilling to undergo a cervical operation, those unable to undergo surgery due to poor general condition, or those with a mismatch between symptoms and radiological diagnosis.

Therefore, we decided to investigate the outcomes of fluoroscopically guided selective nerve root block as a nonsurgical treatment for cervical radiculopathy. In addition, by analyzing the effect of related factors (etiology, sex, and age), we evaluated the usefulness of this technique.

\section{Materials and Methods}

Twenty-eight patients who visited our department from March 2010 to July 2010 due to cervical radicular pain with a single level pathology that did not show symptom improvement after conservative treatment for at least 2 months duration were included in this study. Those with myelopathy symptoms such as hand motor weakness, clumsiness, or gait disturbance were excluded. There were 16 men and 12 women in total. Mean patient age was 58.6 (range, 37 to 72). In terms of etiologies, 19 (68\%) had spondylosis and 9 $(32 \%)$ had cervical disc herniation, and in terms of selective root block locations, 13 (46\%) were performed on C6 roots and $9(32 \%)$ on $\mathrm{C} 7$ roots (Table 1$)$.

Selective cervical root block was performed by a single orthopaedic surgeon wearing a leaded apron, leaded goggles and a thyroid protector to reduce radiation exposure. In addition, the duration of radiation exposure was minimized.
Table 1. Levels of cervical pathology

\begin{tabular}{ccc}
\hline \hline Level & No. of patients & Rate $(\%)$ \\
\hline C4 & 2 & 7 \\
C5 & 3 & 11 \\
C6 & 13 & 46 \\
C7 & 9 & 32 \\
C8 & 1 & 4 \\
\hline
\end{tabular}

For injections, we combined $0.5 \mathrm{ml}$ of triamcinolone acetonide $(40 \mathrm{mg} / \mathrm{ml}$, Triam injection, Dong-kwang Pharm Co., Seoul, Korea) with $2 \mathrm{ml}$ of lebobupivacaine $(2.5 \mathrm{mg} /$ $\mathrm{ml}$, Chirocaine, Abbott laboratories, Elverum, Norway) and $2 \mathrm{ml}$ of saline. A 23 gauge, 3.5 inch spinal needle was used for the procedure, which was performed in a fluoroscopic room without any special anesthetic or stabilizer. To avoid carotid sheath injury, an entry point on the posterior border of the sternocleidomastoid muscle was used. Under realtime fluoroscopic view, the tip of the needle was positioned by checking the antero-posterior and anterior oblique views. The spinal needle trajectory was then decided in anterior oblique view, which allowed the neural foramen to be clearly observed. By checking the antero-posterior view, the needle was engaged and progressed until its tip was placed on half of the lateral mass in the antero-posterior view and superior and posterior to the neural foramen in the oblique view (Fig. 1). After the needle was correctly positioned, less than $1 \mathrm{ml}$ of radiographic contrast medium (Omnipaque
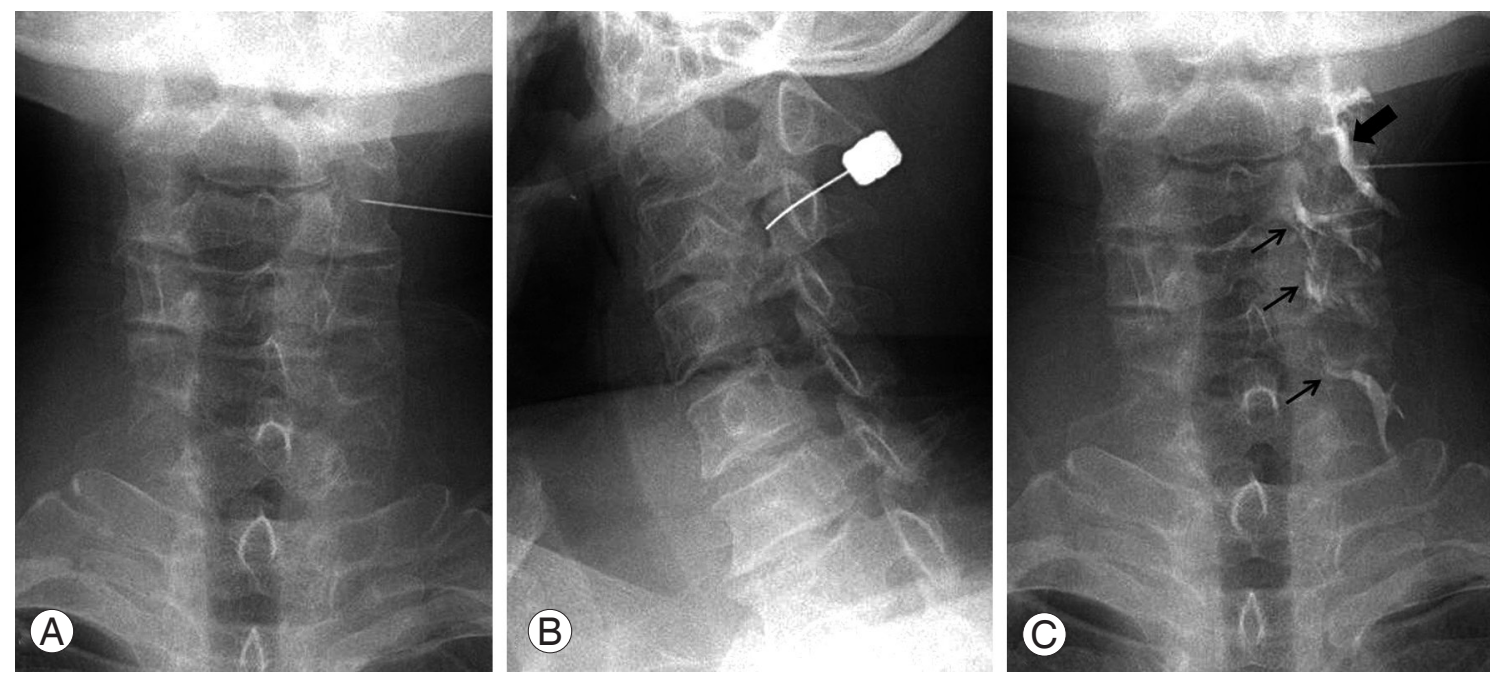

Fig. 1. (A) Antero-posterior radiograph demonstrating a needle in its final position within the left C3-4 intervertebral foramen. The needle tip lies half way between the medial and lateral borders of the lateral mass. (B) Left anterior oblique radiograph demonstrating a needle in position along the posterior aspect of the left C3-4 intervertebral foramen. (C) Antero-posterior radiograph taken after injecting less than $1 \mathrm{cc}$ of radiographic contrast medium. Contrast outlines the exiting nerve root (thick arrow) and extends along the epidural space and below exiting nerve roots (thin arrows) 
Table 2. Categorization of patient satisfaction

\begin{tabular}{ll}
\hline \hline Assignment & \multicolumn{1}{c}{ Patient satisfaction response } \\
\hline Excellent & $\begin{array}{l}\text { Treatment met patient's expectations. } \\
\text { Patient did not improve as he/she has hoped, } \\
\text { but he/she would undergo the same treat- } \\
\text { ment for the same outcome. }\end{array}$ \\
Fair & $\begin{array}{l}\text { The treatment helped, but patient would not } \\
\text { undergo the same treatment for the same } \\
\text { outcome. } \\
\text { Patient was the same or worse than before } \\
\text { treatment. }\end{array}$ \\
\hline
\end{tabular}

300, GE Healthcare, Cork, Ireland) was injected to confirm the targeted cervical root from the spinal cord and appropriate needle positioning.

Nerve root block was performed once fortnightly up to three times. When subjective symptoms improved by more than $90 \%$, the scheduled procedures were cancelled. Clinical assessments were performed using visual analogue scale (VAS) and patient satisfaction scores for procedure outcomes and responses to repeat procedures [4] (Table 2). Relief and the recurrence of symptoms, additional medication history, and management using other conservative methods were evaluated. Assessments were performed before the procedures and at 1 week and at 3, 6, and 12 months after the final procedures.

All patients provided informed consent and the study was approved by our Institutional Review Board.

Statistical analysis was performed using SPSS ver. 18.0 (SPSS, Chicago, IL, USA), and statistical significance was accepted for $p$-values of $<0.05$.

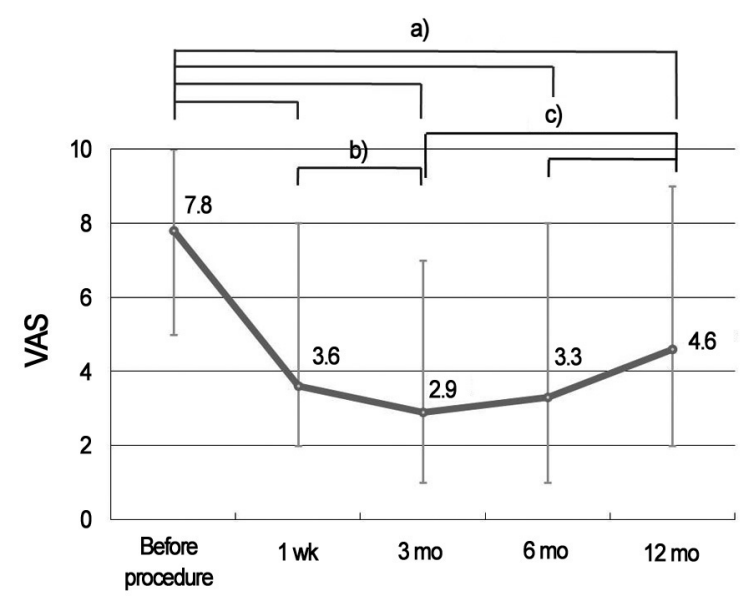

Fig. 2. Graph showing changes in mean visual analogue scale (VAS) scores for cervical radicular pain. ${ }^{\mathrm{a}) \mathrm{b}), \mathrm{c})} p<0.05$.

\section{Results}

The average number of selective nerve root block procedures per patient was 2.8 (range, 1 to 3 ). The average VAS score was 7.8 points (range, 5 to 10 points) preoperatively and this improved to 3.6 points (range, 2 to 8 points) at 1 week, and at 3, 6, and 12 months after the procedures, VAS scores were 2.9 (range, 1 to 7), 3.3 (range, 1 to 8), and 4.6 points (range, 2 to 9 ), respectively. VAS scores significantly improved at all times (Wilcoxon's signed rank test, $p<$ 0.05 ), but showed an increasing trend after 3 months (Fig. 2). However, subgroup analysis revealed no significant VAS score differences with respect to etiology, sex, or age (Mann-Whitney test, $p>0.05$ ) (Table 3).

Before the procedures, all patients were on medication and treated conservatively; medications after the procedures were determined by patient request. Five patients (18\%) requested additional medication at 3 months after the procedures, 8 patients $(29 \%)$ at 6 months, and 13 patients $(46 \%)$ at 12 months. Average symptom-free duration was 7.8 months (range, 1 to 12 months). Patient satisfactory assignments were excellent or good in 20 cases (71\%) at 3 months, in 18 cases (64\%) at 6 months, and in 14 cases (50\%) at 12 months (Fig. 3).

Two patients underwent surgery after the procedures because of recurred radicular pain; both were cervical spondylosis cases. Operations were performed at 8 and 11 months, respectively. In terms of complications, one patient complained of minor causalgia, which was resolved at 5 months, and another developed Horner's syndrome with temporary ptosis, which resolved in a week.

\section{Discussion}

Cervical radiculopathy arises from complex pathophysiologic processes affecting neural elements within the stenotic

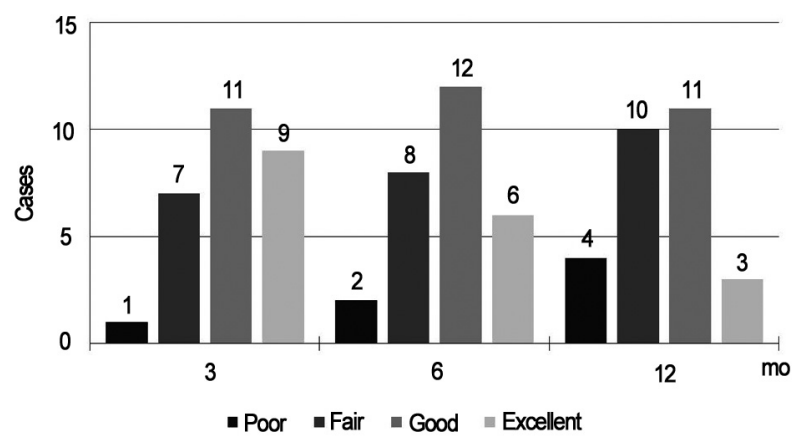

Fig. 3. Box plots showing changes in patient satisfaction after selective nerve root block for cervical radiculopathy. 
Table 3. Statistical analysis of improvements in visual analogue scale scores

\begin{tabular}{lccccc}
\hline \hline \multirow{2}{*}{ Factor } & \multirow{2}{*}{ Before procedure } & \multicolumn{3}{c}{ After procedure } \\
\cline { 3 - 6 } & & At 1 wk & At 3 mo & At 6 mo & At 12 mo \\
\hline Etiology $^{\text {a) }}$ & 0.569 & 0.850 & 0.340 & 0.381 & 0.970 \\
Sex & 0.535 & 0.443 & 0.971 & 0.931 & 0.913 \\
Age $^{\text {b) }}$ & 0.512 & 0.468 & 0.809 & 0.756 & 0.557 \\
\hline
\end{tabular}

Values are presented as $p$-value.

${ }^{\text {a) }}$ Between cases of spondylosis and disc herniation, ${ }^{\text {b) }}$ Between cases $>60$ and $\leq 60$ years old.

foramen. Exiting nerve roots and their nutrient vessels lack a perineurium and have a poorly developed epineurium, and thus, are particularly vulnerable to mechanical and chemical injury [5]. Compression of the venous plexus within the neural foramen can lead to congestion, ischemia, intraneural edema, and increased intraneural pressure [4], and compression of the neural tissue itself may lead to intraneural edema [6]. One probable result of long-standing compressive and mechanical insult to nerve roots is a state of inflammation and fibrosis [5]. Furthermore, in an animal model of radiculopathy induced by inflammatory changes, elevated levels of phospholipase $\mathrm{A}_{2}\left(\mathrm{PLA}_{2}\right)$ were found within nerve roots and the dorsal root ganglion, and additional chemical or inflammatory insult to nerve roots may result from the spillage of inflammogens and neuropeptides from adjacent degenerating cervical structures $[7,8]$.

The role of selective nerve root block has been discussed by many researchers [8-12]. It is generally accepted that the symptomatic relief offered by corticosteroids is a consequence of their potent anti-inflammatory properties $[8,13]$. Furthermore, it has been found that the administration of betamethasone around injured nerve roots can reduce the activity of $\mathrm{PLA}_{2}[8,12,13]$. In addition, it has been suggested that corticosteroids could have a direct anesthetic effect on small unmyelinated nociceptive C-fibers within irritated neural tissue and have anti-inflammatory properties [13-15], and lidocaine may have a therapeutic effect due to its ability to improve blood flow and reduce neural dysfunction in injured nerve roots $[16,17]$.

Published reports describe diverse outcomes after selective nerve root block. Bush et al. [11] in their series of 68 patients, reported that $81 \%$ achieved complete relief from arm and neck pain, although the authors also used other techniques, including a cervical plexus block. Vallée et al. [9] reported a significant reduction in pain in $62 \%$ of 32 patients with cervical radiculopathy, but did not use contrast to confirm the position of the needle tip. Cyteval et al. [17] described a technique conducted with the patient supine on a computed tomography table, and reported that $60 \%$ achieved good pain relief at 6 months. Razzaq et al. [18] reported $28.6 \%$ achieved significant and $14.3 \%$ moderate pain relief at 6 months. Obviously, it is difficult to compare results directly because the amounts of drugs injected and methods of intervention differ, and relatively few studies have followed patients in the long-term [18,19]. Accordingly, this study was undertaken to evaluate pain relief in the long-term (12 months).

Selective nerve root block in the cervical spine has been associated with several severe neurologic complications $[20,21]$. Cerebral infarction is a severe complication, and is considered to be the result of embolization due to particles of injected corticosteroid. For this reason, some doctors prefer betamethasone and dexamethasone to methylprednisolone and triamcinolone, because those agents are manufactured at smaller particle sizes in suspension form. However, in the present study, we used triamcinolone because it is more effective than betamethasone. Spinal cord infarction is suspected to be a result of vascular perforation or direct spinal injury by the needle. Shim et al. [21] reported direct injury of the spinal cord by the needle during nerve root block in the lateral decubitus position. Therefore, to prevent this complication, real time antero-posterior view was used when the needle was applied in the present study. In addition, care was taken to ensure that the tip of the needle was finally located halfway between the medial and lateral border of the lateral mass, as insertion beyond this depth risks puncture of the dural sleeve or thecal sac. Furthermore, in the oblique view, the approach was undertaken via the posterior wall of the foramen, which we considered the safest means of access, and the location of the needle was confirmed by injecting radioactive material before injecting the drug. The prevention of needle tip displacement anteriorly 
is important for two reasons. First, the proximity of the vertebral artery and articular pillars is a potential problem [9]. Second, anterior needle tip displacement can significantly increase the probability of minor complications [21]. In addition, we used the relatively safe nonionic monomer radiographic contrast medium, iohexol (Omnipaque 300), and did not experience any severe nerve damage or complication. Nevertheless, we did experience one case of transient Horner's syndrome due to stellate ganglion paralysis, which resolved in time, and there was one case of causalgia of unidentified pathophysiology, which was considered a type of reflex sympathetic dystrophy.

In the present study, we hypothesized that the effects of cervical nerve root injection with steroid and local anesthetic agents would not provide long-term pain relief in cervical radiculopathy. Five time points were chosen, which included before the procedure, and at 1 week and 3, 6, and 12 months after the procedure, to determine temporal effects. The results showed cervical radicular pain was relieved after the procedure. However, VAS scores showed an increasing tendency after 3 months, and although no significant difference was noted between mean VAS scores at 3 and 6 months, the difference between scores at 6 and 12 months was significant. This suggests that if the benefit of injection is not realized at 6 months, operative intervention should be considered, rather than waiting for a delayed improvement. In the present study, two patients were operated on at 8 and 11 months, respectively. Interestingly, both were spondylosis cases; however, no significant difference was observed between disc herniation and spondylosis cases in terms of pain improvement.

We acknowledge that the sample size of the present study was small. The size of the cohort was limited because of concerns of recall bias and because only a limited number of patients had undergone single level cervical radiculopathy and been managed conservatively for over 2 months. Furthermore, this was an observational study without a comparative treatment arm, and our results could have been affected by the natural history of cervical radicular pain syndrome or by nonspecific treatment effects. A larger-scale prospective study is required to confirm our findings and to determine the long-term therapeutic value of selective nerve root block for cervical radiculopathy.

\section{Conclusions}

Although selective nerve root block for cervical radicu- lopathy is limited as a definitive treatment, it appears to be useful in terms of providing relief from radicular pain in approximately $50 \%$ of patients at 12 months. Furthermore, the procedure could be performed under fluoroscopic guidance without severe complications.

\section{REFERENCES}

1. Radhakrishnan K, Litchy WJ, O'Fallon WM, Kurland LT. Epidemiology of cervical radiculopathy: a population-based study from Rochester, Minnesota, 1976 through 1990. Brain 1994;117:325-35.

2. Lestini WF, Wiesel SW. The pathogenesis of cervical spondylosis. Clin Orthop Relat Res 1989;(239):69-93.

3. Sampath P, Bendebba M, Davis JD, Ducker T. Outcome in patients with cervical radiculopathy. Prospective, multicenter study with independent clinical review. Spine (Phila Pa 1976) 1999;24:591-7.

4. Hoyland JA, Freemont AJ, Jayson MI. Intervertebral foramen venous obstruction. A cause of periradicular fibrosis? Spine (Phila Pa 1976) 1989;14:558-68.

5. Rydevik B, Brown MD, Lundborg G. Pathoanatomy and pathophysiology of nerve root compression. Spine (Phila Pa 1976) 1984;9:7-15.

6. Olmarker K, Rydevik B, Holm S. Edema formation in spinal nerve roots induced by experimental, graded compression: an experimental study on the pig cauda equina with special reference to differences in effects between rapid and slow onset of compression. Spine (Phila Pa 1976) 1989;14:569-73.

7. Rothman SM, Winkelstein BA. Cytokine antagonism reduces pain and modulates spinal astrocytic reactivity after cervical nerve root compression. Ann Biomed Eng 2010;38:2563-76.

8. Slipman CW, Lipetz JS, Jackson HB, Rogers DP, Vresilovic EJ. Therapeutic selective nerve root block in the nonsurgical treatment of atraumatic cervical spondylotic radicular pain: a retrospective analysis with independent clinical review. Arch Phys Med Rehabil 2000;81:741-6.

9. Vallée JN, Feydy A, Carlier RY, Mutschler C, Mompoint $\mathrm{D}$, Vallée CA. Chronic cervical radiculopathy: lateral-approach periradicular corticosteroid injection. Radiology 2001;218:886-92.

10. Windsor RE, Storm S, Sugar R, Nagula D. Cervical transforaminal injection: review of the literature, complications, and a suggested technique. Pain Physician 
2003;6:457-65.

11. Bush K, Chaudhuri R, Hillier S, Penny J. The pathomorphologic changes that accompany the resolution of cervical radiculopathy: a prospective study with repeat magnetic resonance imaging. Spine (Phila Pa 1976) 1997;22:183-6.

12. Lee HM, Weinstein JN, Meller ST, Hayashi N, Spratt KF, Gebhart GF. The role of steroids and their effects on phospholipase A2: an animal model of radiculopathy. Spine (Phila Pa 1976) 1998;23:1191-6.

13. Wilson-MacDonald J, Burt G, Griffin D, Glynn C. Epidural steroid injection for nerve root compression: a randomised, controlled trial. J Bone Joint Surg Br 2005;87:352-5.

14. Johansson A, Hao J, Sjölund B. Local corticosteroid application blocks transmission in normal nociceptive C-fibres. Acta Anaesthesiol Scand 1990;34:335-8.

15. Yabuki S, Kikuchi S. Nerve root infiltration and sympathetic block: an experimental study of intraradicular blood flow. Spine (Phila Pa 1976) 1995;20:901-6.

16. Yabuki S, Kawaguchi Y, Nordborg C, Kikuchi S, Rydevik B, Olmarker K. Effects of lidocaine on nucleus pulposus-induced nerve root injury: a neurophysiologic and histologic study of the pig cauda equina. Spine (Phila Pa 1976) 1998;23:2383-9.

17. Cyteval C, Thomas E, Decoux E, et al. Cervical radiculopathy: open study on percutaneous periradicular foraminal steroid infiltration performed under CT control in 30 patients. AJNR Am J Neuroradiol 2004;25:441-5.

18. Razzaq AA, O'Brien D, Mathew B, Bartlett R, Taylor D. Efficacy and durability of fluoroscopically guided cervical nerve root block. Br J Neurosurg 2007;21:3659.

19. Huston CW, Slipman CW, Garvin C. Complications and side effects of cervical and lumbosacral selective nerve root injections. Arch Phys Med Rehabil 2005;86:277-83.

20. Ma DJ, Gilula LA, Riew KD. Complications of fluoroscopically guided extraforaminal cervical nerve blocks: an analysis of 1036 injections. J Bone Joint Surg Am 2005;87:1025-30.

21. Shim DM, Kim TK, Im JC. Acute spinal cord injury after cervical nerve root block. J Korean Orthop Assoc 2010;45:408-12. 